\title{
BAYESIAN SPATIAL ANALYSIS FOR THE EVALUATION OF BREAST CANCER DETECTION METHODS
}

\author{
Jeff Ching-Fu Hsieh ${ }^{1}$, Susanna M. Cramb ${ }^{2}$, James M. MCGree ${ }^{1, *}$, \\ Peter D. BaAde ${ }^{2}$, Nathan A. M. Dunn ${ }^{3}$ and Kerrie L. Mengersen ${ }^{1}$ \\ Queensland University of Technology
}

\begin{abstract}
Summary
This study investigated the impact of spatial location on the effectiveness of population-based breast screening in reducing breast cancer mortality compared to other detection methods among Queensland women. The analysis was based on linked population-based datasets from BreastScreen Queensland and the Queensland Cancer Registry for the period of 1997 to 2008 for women aged less than 90 years at diagnosis. A Bayesian hierarchical regression modelling approach was adopted and posterior estimation was performed using Markov chain Monte Carlo techniques. This approach accommodated sparse data resulting from rare outcomes in small geographic areas, while allowing for spatial correlation and demographic influences to be included. A relative survival model was chosen to evaluate the relative excess risk for each breast cancer related factor. Several models were fitted to examine the influence of demographic information, cancer stage, geographic information and detection method on women's relative survival. Overall, the study demonstrated that including the detection method and geographic information when assessing the relative survival of breast cancer patients helped capture unexplained and spatial variability. The study also found evidence of better survival among women with breast cancer diagnosed in a screening program than those detected otherwise as well as lower risk for those residing in a more urban or socio-economically advantaged regions, even after adjusting for tumour stage, environmental factors and demographics. However, no evidence of dependency between method
\end{abstract}

\footnotetext{
*Author to whom correspondence should be addressed.

${ }^{1}$ Queensland University of Technology (QUT) GPO Box 2434, Brisbane Qld 4001, Australia.

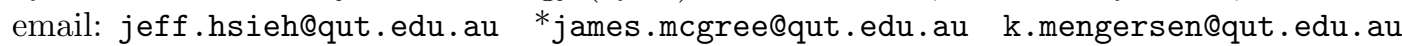
${ }^{2}$ Cancer Council Queensland (CCQ) PO Box 201, Spring Hill Qld 4004, Australia. email: susannacramb@cancerqld.org.au peterbaade@cancerqld.org.au

${ }^{3}$ BreastScreen Queensland (BSQ), Preventive Health Unit, Department of Health PO Box 2368 Fortitude Valley BC Qld 4006, Australia. email: Nathan_Dunn@health.qld.gov.au Acknowledgments. This project was supported by an Australian Research Council (ARC) Linkage Project between QUT, CCQ and BSQ. Peter Baade is funded by an National Health and Medical Research (NHMRC) Career Development Fellowship.
} 


\section{BAYESIAN SPATIAL MODEL FOR BREAST CANCER}

of detection and geographic location was found. This project provides a sophisticated approach to examining the benefit of a screening program while considering the influence of geographic factors.

Key words: Bayesian modelling; breast screening; Markov chain Monte Carlo; relative excess risk; relative survival; spatial variability.

\section{Introduction}

Breast cancer is the most common invasive cancer among Australian women, accounting for an estimated $27 \%$ (or 14,560) of all cancers diagnosed among women in 2012 and is the second leading cause of cancer death in Australia (2,840 deaths, or $15 \%$ of cancer deaths in 2010) (AIHW \& AACR, 2012). Due to the strong relationship between breast cancer stage and survival (AIHW \& NBCC, 2007; Baade et al., 2011a), detection of breast cancer earlier in its disease pathway increases the opportunity for effective treatment to reduce the morbidity associated with this disease, and thus improve long-term prognosis. Mammography screening plays an important role in the early detection of cancer, and has been shown to improve the chance of survival (Berry et al., 2005; Shen et al., 2005).

In a climate of continuing controversy about the value of mammographic screening for reducing breast cancer mortality (McPherson et al., 1997; Olsen \& Gtzsche, 2001; Wishart et al., 2008), there has been strong interest in assessing the efficacy of screening programs to guide appropriate allocation of health care resources (Bordas et al., 2007; Hegarty et al., 2010; Wu et al., 2010). Studies over the past decade have examined the efficacy of breast cancer screening in relation to the prognosis, pathology and survival of breast cancer patients, compared against other methods of detection, and have found method of detection to be an important prognostic factor with women participating in a screening program generally having more favourable tumour stage and better survival than those detected otherwise (McPherson et al., 1997; Shen et al., 2005; Mook et al., 2011; Nagtegaal et al., 2011; Nickson et al., 2012).

Women whose breast cancer is diagnosed through a breast screening program tend to have better prognosis than those diagnosed symptomatically primarily because they are detected at an earlier, less advanced stage. Women with screen detected tumours are more likely to live longer and have longer time to distant recurrence than those detected otherwise (Albert et al., 1978; Morrison, 1985; Kramer et al., 1999; Otto et al., 2003; Tabar et al., 2003; Joensuu et al., 2004; Shen et al., 2005; Bordas et al., 2007; Wishart et al., 2008; Mook et al., 2011). There is a suggestion that at least some of this advantage is due to lead-time and length bias, in which earlier stage tumours and those less aggressive, slow growing tumours, are more likely to be detected by screening programs (Chu et al., 1988; Connor et al., 1989; Shen et al., 2005).

Previous studies have provided strong evidence of geographical variation in female breast cancer incidence and survival with women living in more disadvantaged or rural areas tending to have lower survival (Robsahm \& Tretli, 2008; Huang et al., 2009; Sariego, 2009; Baade et al., 2011a; Cramb et al., 2011a; Dasgupta et al., 2012; Cramb et al., 2012), even after adjusting for spread of disease at diagnosis (Dasgupta et al., 


\section{BAYESIAN SPATIAL MODEL FOR BREAST CANCER}

2012). However these analyses provide little understanding about how the impact of mammography screening on breast cancer survival varies across geographical location and by demographic sub-groups. This is of particular interest for remote Australians whose access to screening programs and treatment facilities is often limited by the barrier of distance.

A Swedish study (Bordas et al., 2007) investigating this topic was limited due to the homogeneous socio-economic characteristics of the study area. Studies such as these have also been constrained by the difficulty of dealing with sparse data typically associated with spatial data. Recently, Bayesian models and Markov chain Monte Carlo (MCMC) algorithms have been used to accommodate sparse spatial data and to create disease maps to assess the spatial and possible temporal effects associated with the disease of interest (Waller et al., 1997; Ghosh et al., 1999; Osnes \& Aalen, 1999; Lawson et al., 2000; Militino et al., 2001; Lawson, 2001; MacNab \& Dean, 2002; MacNab, 2004; Richardson et al., 2006; Hegarty et al., 2010; Wu et al., 2010; Saez et al., 2012).

The use of relative survival is generally preferred in survival analyses of populationbased disease registry data. Relative survival compares the observed mortality among study subjects (in particular, cancer patients) to the expected mortality based on mortality in their general population counterparts. While the theory of Bayesian univariate spatial models has been extensively researched, there are few examples of Bayesian relative survival spatial models in the literature. Only Fairley et al. (2008), Cramb et al. (2011a) and Saez et al. (2012) have utilized additional spatial information from the neighbouring regions, thus facilitating the description of possible spatial correlation and improved estimates of relative survival cancer outcomes. To our knowledge, ours is the first study to examine the impact of geographical location on the effectiveness of breast cancer screening using a fully Bayesian spatial relative survival framework.

This study applies Bayesian spatial hierarchical relative survival models to investigate the impact of spatial location on the effectiveness of population-based breast screening in reducing breast cancer mortality, after accounting for the influence of demographics and clinical features. Specifically, this paper aims to answer the following questions:

Q1: How do breast cancer patients' demographics, tumour stage, socio-economic status and geographic remoteness influence their relative survival?

Q2: How do the screening programs influence the relative survival of breast cancer patients, taking into account patients' demographics, tumour stage, socio-economic status and geographic remoteness?

Q3: Are there substantive interactions between the screening program and the patients' socio-economic status and geographic remoteness? 


\section{Methods}

\subsection{Data sources}

Data were extracted from the BreastScreen Queensland (BSQ) and Queensland Cancer Registry (QCR) datasets. BreastScreen Queensland is part of the BreastScreen Australia Program which was established in 1991 by the Australian Government and the State and Territory governments. It is a public health cancer screening program that provides the only population-based breast cancer screening service in Queensland. More than 202,000 women were screened by BSQ during 2007. The Queensland Cancer Registry is a population-based registry for which notification of invasive cancers is required by law. Between 1997 and 2007, the QCR recorded over 24,000 cases of invasive breast cancer among women. Data from the QCR were linked to the BSQ data by BSQ staff using a deterministic matching process with over $90 \%$ completeness of matching. All invasive breast cancers (ICD-O-3 code $=\mathrm{C} 50)$ diagnosed between 1 January 1997 and 31 December 2007, among Queensland women aged less than 90 years at diagnosis, were eligible for inclusion in this study. Follow-up was up to 31 December 2008.

\subsection{Explanation of variables}

The data which were analyzed in the modelling process included observed mortality, age group at diagnosis (0-39 years, 40-49, 50-59, 60-69 and 70-89 years), marital status at time of diagnosis (married, never married, widowed/divorced/separated or unknown), indigenous status (Indigenous, non-Indigenous and unknown), tumour stage at diagnosis, detection method and geographic location and classification. As the study includes individual-level and area-level variables, the deaths among breast cancer patients living in each SLA were aggregated by each individual-level variable categories. For example, the deaths among breast cancer patients living in a specific Statistical Local Area (SLA), of a specific age group, with the tumour diagnosed at certain stage and method etc. were aggregated together.

Stage at diagnosis was classified into groups of localised, advanced and unknown using information on tumour diameter and lymph-node involvement collected by the QCR (Baade et al., 2011a).

Standard BSQ definitions (provided by BSQ staff) were used to categorise the method of detecting breast cancer into screen detected, interval detected and nonscreen detected. Screen detected breast cancers are those diagnosed through a systematic method (e.g. mammography) during a regular BreastScreen Australia screening episode. An interval breast cancer is any invasive breast cancer diagnosed in the 24month interval following a negative screening episode and before the next scheduled screening examination (Kavanagh et al., 1999). A non-screen detected breast cancer is defined as either (i) any invasive breast cancer diagnosed prior to the commencement of screening or an invasive breast cancer diagnosed outside of the screening program after 24 months have elapsed following a negative screening episode; (ii) an invasive breast cancer diagnosed in a woman who had not participated in an organised screening program. 
Geographical location was defined by Statistical Local Areas (SLAs), which are spatial units likely to be socially and economically relevant to their residents, using the 2006 version of the Australian Standard Geographical Classification (ASGC). In 2006, there were 478 SLAs in Queensland with a median population of 5,810.

Geographic remoteness of residence was categorised by an Accessibility-Remoteness Index of Australia Plus (ARIA+) classification, with the categories of Major City, Inner Regional, Outer Regional, Remote and Very Remote areas. Due to small numbers Remote and Very Remote categories were combined. These classifications are based on road distance from a locality to the closest service center (AIHW, 2004).

Socio-economic status (SES) measured by Socio-Economic Indexes for Area (SEIFA) is based on the Index of Relative Socio-economic Advantage and Disadvantage (IRSAD) calculated by the Australian Bureau of Statistics (Australian Bureau of Statistics, 2008). This index was chosen because it does not include Indigenous status in its derivation. It provides a general measure of socio-economic disadvantage, with its scores collapsed into quintiles Q1 (Most disadvantaged) to Q5 (Most advantaged).

Population all-cause age-specific mortality rates were calculated from the unit record mortality file for Queensland residents, and generated for each combination of SLA, gender and broad time period (1997-2002; 2003-2007). To provide greater stability, mortality and population data for each SLA were combined with the relevant data from the neighbouring SLAs, as defined by the adjacency matrix. The expected number of deaths due to any cause among the breast cancer cohort for each combination of variables was generated from these population mortality rates. Although these expected deaths include the effect of deaths due to breast cancer, this does not, in practice, affect the estimated survival proportions (Dickman et al., 2004).

\subsection{Model specification}

Following others such as Dickman et al. (2004) and Cramb et al. (2011b), and consistent with most modern survival analyses on population-based cancer data, a relative survival model was used. This has the advantage of not requiring cause of death information among the breast cancer cohort (Dickman et al., 2004; Pohar \& Stare, 2006; Fairley et al., 2008; Cramb et al., 2011b).

To model the observed number of deaths a generalized linear model with a Poisson assumption was used.

$$
d_{\epsilon} \sim \operatorname{Poisson}\left(\mu_{\epsilon}\right)
$$

where $d_{\epsilon}$ is the observed number of deaths due to any cause among the cohort of breast cancer patients for each stratum $(\epsilon)$, and $\mu_{\epsilon}$ is the mean of the observed deaths due to any cause.

For a relative survival model that measures the relative excess risk (RER), the mean of the observed deaths $\left(\mu_{\epsilon}\right)$ can be modelled by

$$
\mu_{\epsilon}=d_{\epsilon}^{*}+y_{\epsilon} \times \exp \left(\eta_{\epsilon}\right)
$$

where $d_{\epsilon}^{*}$ is the expected number of non-breast cancer deaths among the cohort of breast cancer patients for each stratum $(\epsilon)$. The person-time at risk, $y_{\epsilon}$, is the total 


\section{BAYESIAN SPATIAL MODEL FOR BREAST CANCER}

amount of time in each year that the study subjects are at risk of dying from breast cancer, and $\eta_{\epsilon}$ represents the excess hazard. The subscript $\epsilon$ is the general indicator for the partition of data including, as appropriate, time $t$, the spatial unit $i$, and the covariates described in Section 2.2 (and shown in Table 1).

The exponentiated values of the individual $\eta_{\epsilon}$ components provide the relative excess risk for the corresponding model variables (e.g. $\exp (\alpha), \exp (\beta)$ and $\left.\exp \left(u_{i}+v_{i}\right)\right)$. The excess hazard $\left(\eta_{\epsilon}\right)$ is estimated by

$$
\eta_{\epsilon}=\alpha_{t}+\boldsymbol{\beta} \boldsymbol{x}+u_{i}+v_{i}
$$

where $\boldsymbol{\beta}$ is the coefficient vector associated with the vector of predictor variables $\boldsymbol{x}$ listed in Section 2.2 as well as interactions between detection method and geographic location; $\alpha_{t}$ is the $t^{t h}$ intercept that varies by follow-up interval (i.e. number of years from diagnosis to death or censoring) for $t=1,2, \ldots, 12$; and $u_{i}$ and $v_{i}$ represent the $i^{\text {th }}$ SLA $(i=1,2, \ldots, 478)$ with spatially structured and unstructured random effects, respectively. The prior distribution for all entries of $\boldsymbol{\beta}$ and all $\alpha_{t}$ is a zero mean Normal distribution with a Gamma $(0.5,0.005)$ hyperprior distribution for the precision; $v_{i}$ is the geographic unstructured random effect and also has a zero mean Normal prior distribution with a Gamma(0.5,0.005) hyperprior distribution for the precision; and $u_{i}$ is the geographic structured random effect which has a conditional autoregressive (CAR) prior distribution (Besag et al., 1991; Bell \& Broemeling, 2000; Banerjee et al., 2003; Best et al., 2005; Earnest et al., 2007; Wakefield, 2007). A CAR prior for $u_{i}$ is of the form

$$
\begin{gathered}
u_{i} \mid u_{(-i)} \sim \operatorname{Normal}\left(\frac{\sum_{j} w_{i j} u_{j}}{w_{i+}}, \frac{\sigma_{u}^{2}}{w_{i+}}\right) \\
w_{i+}=\sum_{j} w_{i j},
\end{gathered}
$$

where $u_{(-i)}$ denotes the value of $u_{i}$ in all areas except the $i^{\text {th }}$ area. The $w_{i j}$ is the $i j^{\text {th }}$ element of a symmetric $n \times n$ 'weight' matrix $\boldsymbol{W}$ with diagonal elements $w_{i i} \equiv 0$. We choose $w_{i j}=1$ if locations $i$ and $j$ are neighbours and $w_{i j}=0$ otherwise, hence $w_{i+}$ is equal to the number of neighbours. The neighbourhood matrices of Queensland by Cramb et al. (2011b) were applied to the CAR prior distribution in our models.

\subsection{Computation}

The Bayesian hierarchical models were estimated using $\mathrm{R}$ ( $\mathrm{R}$ Development Core Team, 2011) and WinBUGS (Lunn et al., 2000). The R program was used to handle data manipulation prior to running the model, and to analyse the posterior densities of the model parameters. WinBUGS was used to perform MCMC to produce the posterior samples. Each model was run with 2 chains until convergence (total iterations ranged from 100,000 to 150,000) and 30,000 simulated samples were retained for inference.

Convergence checks for model parameters were employed in $\mathrm{R}$ by means of Gelman \& Rubin (1992) convergence diagnostic, trace plots, density plots and autocorrelation plots. 


\section{BAYESIAN SPATIAL MODEL FOR BREAST CANCER}

\subsection{Model fitting and evaluation}

Models were fitted to answer the three research questions. In order to answer the first question (Q1), three models were fitted (M1_1, M1_2 \& M1_3), each including the demographic and stage variables. In addition, Model 1 included remoteness, Model 2 included area disadvantage and Model 3 included both remoteness and disadvantage. For the second question (Q2), the models from question 1 (M1_1 to M1_3) were refitted with the addition of detection method (M2_1 to M2_3). For the last question (Q3), the M2 models were extended by adding the interaction between the detection method and the geographic variables to each model (M3_1 to M3_3). Several other models were fitted outside the scope of the three questions. One such model, which consisted of all the demographic, stage and detection method variables (M0_2), was used to assess the spatial variability or inequalities of relative survival among breast cancer patients in Queensland.

The posterior predictive check (PPC) (Gelman et al., 2004) was used to assess how adequately the model represents the observed data. The assessment procedure is as follows. First, the expected value $\mu$ is calculated using the posterior distribution of relevant parameters. Second, the expected data $d$ are generated from a Poisson distribution using the estimated $\mu$, and a posterior predictive distribution is formed. Third, each observed $d$ is then compared with the corresponding posterior predictive distribution to assess whether it is encompassed in the $95 \%$ credible interval (CI) of the distribution. An adequate model is often declared if around 95\% of the observed data are within the $95 \%$ CI of the posterior predictive distribution.

The Deviance Information Criterion (DIC) was used to determine the preferred model, with lower values indicating a 'better' model (Gelman et al., 2004). DIC is calculated as the sum of the mean deviance and an estimate of the effective number of parameters $(\mathrm{pD})$, with smaller deviance indicating better fit, and smaller $\mathrm{pD}$ values indicating less complexity in the fitted model.

The posterior estimate of a relative survival model parameter is declared to be "sufficiently specific" if the $95 \%$ CI of the corresponding exponentiated regression coefficient does not include the value "1" (Wakefield, 2007; Wu et al., 2010; PuigpinsRiera et al., 2011).

To assess the spatial variability within the model, disease maps of SLA-specific RER were generated and a numerical measure was calculated using the variance of the spatial random effects. The proportion of extra spatial variability was calculated as the ratio of the marginal spatial structured variance to the sum of the variance of both marginal spatial structured and unstructured random effects (Eberly \& Carlin, 2000; Saez et al., 2012).

\section{Results}

Table 1 gives the number of eligible women living in Queensland who had been diagnosed with breast cancer between 1997 and 2007, as well as the percentage of this cohort who had died from all causes up to December $31^{\text {st }} 2008$. 


\section{BAYESIAN SPATIAL MODEL FOR BREAST CANCER}

Table 1: Number of eligible Queensland females diagnosed with breast cancer (19972007) and the percentage of those who died from any cause (1997-2008)

\begin{tabular}{lrr}
\hline & $\begin{array}{r}\text { All BC } \\
\text { incidence } \\
(\mathbf{1 9 9 7 - 2 0 0 7 )}\end{array}$ & $\begin{array}{r}\text { All causes } \\
\text { death (\%) } \\
(\mathbf{1 9 9 7 - 2 0 0 8 )}\end{array}$ \\
\hline \hline All Queensland women (0-89 years at diagnosis) & 23766 & 19.5 \\
ARIA+ & & \\
Major City & 14464 & 19.0 \\
Inner Regional & 5066 & 20.1 \\
Outer Regional & 3351 & 20.3 \\
Remote/Very Remote & 885 & 22.9 \\
SES IRSAD & & \\
Quintile 1 Most disadvantaged & 2871 & 22.2 \\
Quintile 2 & 5179 & 21.5 \\
Quintile 3 & 6204 & 19.7 \\
Quintile 4 & 5638 & 18.5 \\
Quintile 5 Most advantaged & 3874 & 16.2 \\
Detection Method & & \\
Screening & 6902 & 10.0 \\
Interval & 2843 & 14.5 \\
Non-screen & 14021 & 25.2 \\
Age (years) & & \\
0-39 & 1428 & 16.4 \\
40-49 & 4669 & 11.5 \\
50-59 & 6443 & 12.4 \\
60-69 & 5545 & 16.1 \\
70-89 & 5681 & 38.3 \\
Indigenous Status & & \\
Non-Indigenous & 20529 & 21.7 \\
Indigenous & 257 & 31.5 \\
Indigenous Status Unknown & 2980 & 3.8 \\
Marital Status & & \\
Married & 14801 & 14.5 \\
Never Married & 1441 & 21.3 \\
Widowed/Divorced/Separated & 6787 & 31.7 \\
Marital Status Unknown & 737 & 6.1 \\
Tumour Stage & & \\
Localised & 11517 & 10.8 \\
Advanced & 10699 & 23.7 \\
Stage Unknown & 1550 & 55.4 \\
\hline
\end{tabular}

The proportions of women who died from any cause during the study period were larger for women who resided in Remote or Very Remote areas, and those who lived in SLAs with a socio-economic status of quintile 1 or 2 (disadvantaged). The largest proportions of all cause mortality were observed in females whose breast cancer was detected outside a screening program (non-screen), women aged 70-89, Indigenous, 


\section{BAYESIAN SPATIAL MODEL FOR BREAST CANCER}

Table 2: RER of detection methods, demographic, tumour stage and geographic status for aged 0-89 Queensland female residents with breast cancer, 1997-2008

\begin{tabular}{|c|c|c|c|}
\hline \multirow[b]{2}{*}{ Factors } & \multicolumn{3}{|c|}{ RER $[95 \%$ CI] } \\
\hline & Model M0_2 & Model M1_1 & Model M2_3 \\
\hline \multicolumn{4}{|l|}{ ARIA+ } \\
\hline Major City & - & 1.00 & 1.00 \\
\hline Inner Regional & - & $1.1208[0.9643,1.2710]$ & $1.0957[0.9578,1.2420]$ \\
\hline Outer Regional & - & $1.1528[0.8953,1.3530]$ & $1.1393[0.9562,1.3270]$ \\
\hline Remote/Very Remote & - & $1.4065[1.0370,1.7530]$ & $1.3712[1.0720,1.6980]$ \\
\hline \multicolumn{4}{|l|}{ SES IRSAD } \\
\hline Quintile 1 Most disadvantaged & - & - & $1.2060[0.9971,1.4430]$ \\
\hline Quintile 2 & - & - & $1.1519[0.9770,1.3550]$ \\
\hline Quintile 3 & - & - & $1.1698[1.0030,1.3610]$ \\
\hline Quintile 4 & - & - & $1.0928[0.9367,1.2710]$ \\
\hline Quintile 5 Most advantaged & - & - & 1.00 \\
\hline \multicolumn{4}{|l|}{ Detection Method } \\
\hline Screening & $0.4136[0.3545,0.4777]$ & - & $0.4116[0.3531,0.4742]$ \\
\hline Interval & $0.7033[0.6102,0.8060]$ & - & $0.7014[0.6087,0.7989]$ \\
\hline Non-screen & 1.00 & - & 1.00 \\
\hline \multicolumn{4}{|l|}{ Age (years) } \\
\hline $0-39$ & $0.9159[0.7776,1.0690]$ & $1.1563[0.9821,1.3490]$ & $0.9127[0.7740,1.0630]$ \\
\hline $40-49$ & $0.8243[0.7273,0.9322]$ & $0.9128[0.8046,1.0320]$ & $0.8230[0.7241,0.9291]$ \\
\hline $50-59$ & 1.00 & 1.00 & 1.00 \\
\hline $60-69$ & $1.1332[0.9979,1.2780]$ & $1.1244[0.9901,1.2710]$ & $1.1282[0.9930,1.2720]$ \\
\hline $70-89$ & $1.4869[1.3140,1.6770]$ & $1.6122[1.4250,1.8140]$ & $1.4835[1.3170,1.6720]$ \\
\hline \multicolumn{4}{|l|}{ Indigenous Status } \\
\hline Non-Indigenous & 1.00 & 1.00 & 1.00 \\
\hline Indigenous & $1.8588[1.4050,2.3840]$ & $1.8402[1.3860,2.3500]$ & $1.7540[1.3250,2.2390]$ \\
\hline Indigenous Status Unknown & $0.0352[0.0087,0.0710]$ & $0.0374[0.0099,0.0754]$ & $0.0420[0.0145,0.0785]$ \\
\hline \multicolumn{4}{|l|}{ Marital Status } \\
\hline Married & 1.00 & 1.00 & 1.00 \\
\hline Never Married & $1.2791[1.0890,1.4830]$ & $1.3140[1.1190,1.5310]$ & $1.2878[1.0990,1.4920]$ \\
\hline Widowed/Divorced/Separated & $1.3924[1.2660,1.5270]$ & $1.4134[1.2860,1.5490]$ & $1.3922[1.2640,1.5260]$ \\
\hline Marital Status Unknown & $0.4181[0.1836,0.7238]$ & $0.4159[0.1838,0.7172]$ & $0.4269[0.2031,0.7168]$ \\
\hline \multicolumn{4}{|l|}{ Tumour Stage } \\
\hline Localised & 1.00 & 1.00 & 1.00 \\
\hline Advanced & $4.1156[3.6040,4.7000]$ & $4.5599[3.9830,5.2240]$ & $4.0447[3.5230,4.6150]$ \\
\hline Stage Unknown & $13.612[11.690,15.770]$ & $17.033[14.600,19.890]$ & $13.358[11.470,15.520]$ \\
\hline Spatial Structured Variance & $0.0215[0.0086,0.0406]$ & $0.0070[0.0009,0.0359]$ & $0.0048[0.0012,0.0155]$ \\
\hline Spatial Unstructured Variance & $0.0070[0.0014,0.0219]$ & $0.0077[0.0017,0.0227]$ & $0.0079[0.0016,0.0237]$ \\
\hline Extra Spatial Variability & 0.7603 & 0.4387 & 0.3982 \\
\hline DIC & 35457.2 & 35641.1 & 35458.0 \\
\hline pD & 55.8 & 49.8 & 53.9 \\
\hline PPC & 0.9853 & 0.9858 & 0.9855 \\
\hline
\end{tabular}

widowed/divorced/separated as well as unknown tumour stage.

\subsection{Impact of patient demographics, stage and geographic location (Q1)}

The selection of the final model was based on the effect of remoteness and area disadvantage. The final DIC values (with $\mathrm{pD}$ in brackets) for the three fitted models (M1_1, M1_2 \& M1_3) were 35641.1 (49.8), 35643.8 (53.3) and 35644.1 (53.5) respectively. Based on this, the final model (Table 2) was chosen to be the one that retained remoteness but excluded area disadvantage (M1_1). There was very little difference in the RER estimates for the common variables across the three models (results not shown). Generally the relative excess risk increased for women living in rural/remote 


\section{BAYESIAN SPATIAL MODEL FOR BREAST CANCER}

Table 3: RER of interaction between detection methods and geographic factors for eligible Queensland female residents with breast cancer, 1997-2008

\begin{tabular}{|c|c|c|c|}
\hline Model M3_1 & \multicolumn{3}{|c|}{ RER $[95 \% \mathrm{CI}]$} \\
\hline Factors & Main Effect & Screening Interaction & Interval Interaction \\
\hline \multicolumn{4}{|l|}{ ARIA+ } \\
\hline Major City & 1.00 & 1.00 & 1.00 \\
\hline Inner Regional & $1.1226[0.9714,1.2810]$ & $1.0336[0.7188,1.4200]$ & $1.0376[0.7406,1.3970]$ \\
\hline Outer Regional & $1.2471[1.0150,1.4590]$ & $0.7671[0.4827,1.1200]$ & $0.7867[0.5172,1.1330]$ \\
\hline Remote/Very Remote & $1.4252[1.0790,1.7980]$ & $1.3027[0.7101,2.0980]$ & $0.8675[0.4358,1.4570]$ \\
\hline \multicolumn{4}{|l|}{ SES IRSAD } \\
\hline Quintile 1 Most disadvantaged & - & - & - \\
\hline Quintile 2 & - & - & - \\
\hline Quintile 3 & - & - & - \\
\hline Quintile 4 & - & - & - \\
\hline Quintile 5 Most advantaged & - & - & - \\
\hline \multicolumn{4}{|l|}{ Detection Method } \\
\hline Screening & $0.4218[0.3460,0.5058]$ & - & - \\
\hline Interval & $0.7340[0.6020,0.8780]$ & - & - \\
\hline Non-screen & 1.00 & - & - \\
\hline \multicolumn{4}{|l|}{ Age (years) } \\
\hline $0-39$ & $0.9151[0.7752,1.0660]$ & - & - \\
\hline $40-49$ & $0.8256[0.7283,0.9334]$ & - & - \\
\hline $50-59$ & 1.00 & - & - \\
\hline $60-69$ & $1.1355[1.0010,1.2820]$ & - & - \\
\hline $70-89$ & $1.4956[1.3240,1.6830]$ & - & - \\
\hline \multicolumn{4}{|l|}{ Indigenous Status } \\
\hline Non-Indigenous & 1.00 & - & - \\
\hline Indigenous & $1.7885[1.3640,2.2970]$ & - & - \\
\hline Indigenous Status Unknown & $0.0436[0.0161,0.0789]$ & - & - \\
\hline \multicolumn{4}{|l|}{ Marital Status } \\
\hline Married & 1.00 & - & - \\
\hline Never Married & $1.2926[1.1010,1.4990]$ & - & - \\
\hline Widowed/Divorced/Separated & $1.3957[1.2700,1.5270]$ & - & - \\
\hline Marital Status Unknown & $0.4390[0.2093,0.7413]$ & - & - \\
\hline \multicolumn{4}{|l|}{ Tumour Stage } \\
\hline Localised & 1.00 & - & - \\
\hline Advanced & $4.0809[3.5720,4.6740]$ & - & - \\
\hline Stage Unknown & $13.457[11.570,16.690]$ & - & - \\
\hline Spatial Structured Variance & & $0.0060[0.0012,0.0202]$ & \\
\hline Spatial Unstructured Variance & & $0.0075[0.0016,0.0209]$ & \\
\hline Extra Spatial Variability & & 0.4344 & \\
\hline DIC & & 35462.5 & \\
\hline $\mathrm{pD}$ & & 56.3 & \\
\hline PPC & & 0.9855 & \\
\hline
\end{tabular}

areas, oldest age group (70-89), Indigenous women, those who were not married at time of diagnosis, and those with an advanced or unknown stage tumour.

\subsection{Impact of detection method after adjusting for other fac- tors (Q2)}

The inclusion of detection method provided substantial improvement to the model fit with much smaller DIC values, as evidenced by models M0_2 and M2_3 in Table 2. The inclusion of geographic remoteness and disadvantage in model M0_2 generated three models with similar DIC values that differ by less than 5 (M2_1(remoteness): 
35455.4 (pD 48.8), M2_2(disadvantage): 35457.7 (57.3), M2_3(remoteness \& disadvantage): $35458.0(53.9))$. The model including both geographic remoteness and disadvantage (M2_3) was selected to be the most appropriate model as it provided additional useful information (Table 2). Although models M0_2 and M2_3 had similar DIC and pD values, due to the additional geographical information model M2_3 was preferred over M0_2. The only significant change after including detection method was a reduction in RER among those aged 40-49 years. There remained higher relative excess risk for breast cancer patients residing in remote areas, disadvantaged regions, and those not participating in a screening program.

\subsection{Evidence for interactions (Q3)}

Based on the DIC values (M3_1: 35462.5 (pD 56.3), M3_2: 35466.1 (62.4), M3_3: $35474.2(70.4)$ ), the preferred model with interactions included area remoteness, but not socio-economic status (Table 3 ). There was no visible change to the main effect results after including interaction terms. As interactions between the detection method and area remoteness fluctuated around the baseline value and were not sufficiently specific, a main effects only model was preferred over the interaction model.

\subsection{Spatial survival inequalities}

Figure 1 presents a map of RER (divided into quintiles) in each SLA in Queensland and, in finer detail, the more populated southeast corner of the state, compared to the Queensland average RER (one), before including geographic remoteness and disadvantage (model M0_2). There was evidence of spatial survival inequalities across Queensland SLAs (extra spatial variability $=0.76$ ) with higher relative excess risk in northern and western Queensland and decreased relative excess risk of death in southeast Queensland. In Brisbane, smaller SLA-specific RER values were observed north of the Brisbane river and higher values south of the river. After including geographic remoteness and disadvantage in the analysis (model M2_3), Figure 2 (using the same RER quintile categories as model M0_2) demonstrates a visible reduction in spatial survival inequalities (extra spatial variability $=0.40$ ).

\section{Discussion}

By applying a Bayesian spatial relative survival model to a large population-based cohort of Queensland women diagnosed with breast cancer, we have reinforced recent findings showing that participating in mammographic screening is associated with better breast cancer survival (Shen et al., 2005; Berry et al., 2005, 2006; Bordas et al., 2007; Wishart et al., 2008; Wu et al., 2010; Mook et al., 2011; Nagtegaal et al., 2011; Nickson et al., 2012; Roder \& Olver, 2012). Importantly, this study demonstrated that there was no discernible evidence that this impact of breast cancer screening on survival varied by the women's geographical location, even after accounting for the extent of small area variation, tumour stage at diagnosis and other important demographic variables. 


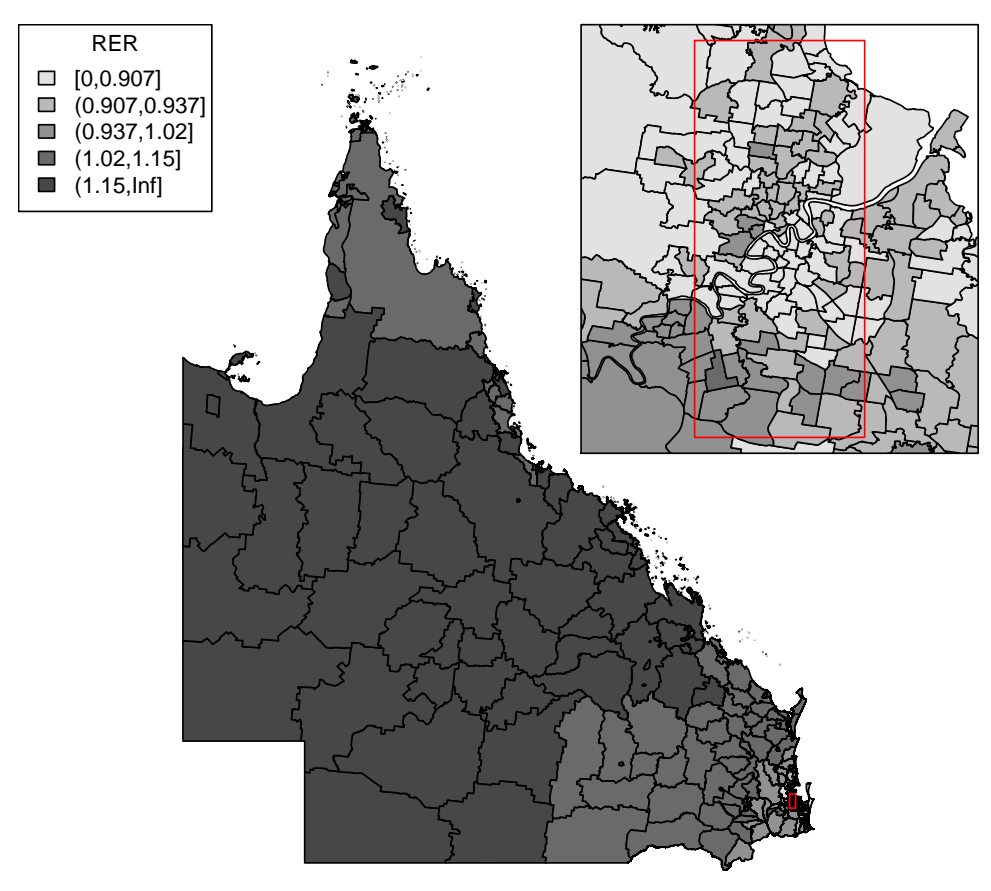

Figure 1: Posterior estimates of RER for each SLA in Queensland \& the southeast corner, based on Model M0_2

The survival advantage that we found for screen-detected cancers does need to be considered in light of the propensity for overdiagnosis in screening programs, in that asymptomatic cancers detected through population screening may not progress to cause symptoms, morbidity or subsequent death, and so would artificially increase the observed survival (Wishart et al., 2008; Bell \& Burton, 2012; Barratt \& Glasziou, 2012). However we found that the survival benefit in participating a screening program was only marginally reduced after adjusting for tumour stage at diagnosis, demonstrating that earlier diagnosis did not explain the survival differential. While we have adjusted for a broad measure of tumour stage at diagnosis, there remains the possibility that some lead-time bias may be unaccounted by not having more detailed stage information. In addition, the lack of clinical information on the aggressiveness of the cancer restricted our ability to completely adjust for the effect of length bias, which reflects the propensity for screening programs to detect a disproportional number of slowly growing tumours (Shen et al., 2005). Thus it is possible that some of the observed association between screening and survival in our study could be explained by residual length bias, or, to a smaller extent, lead-time bias. 


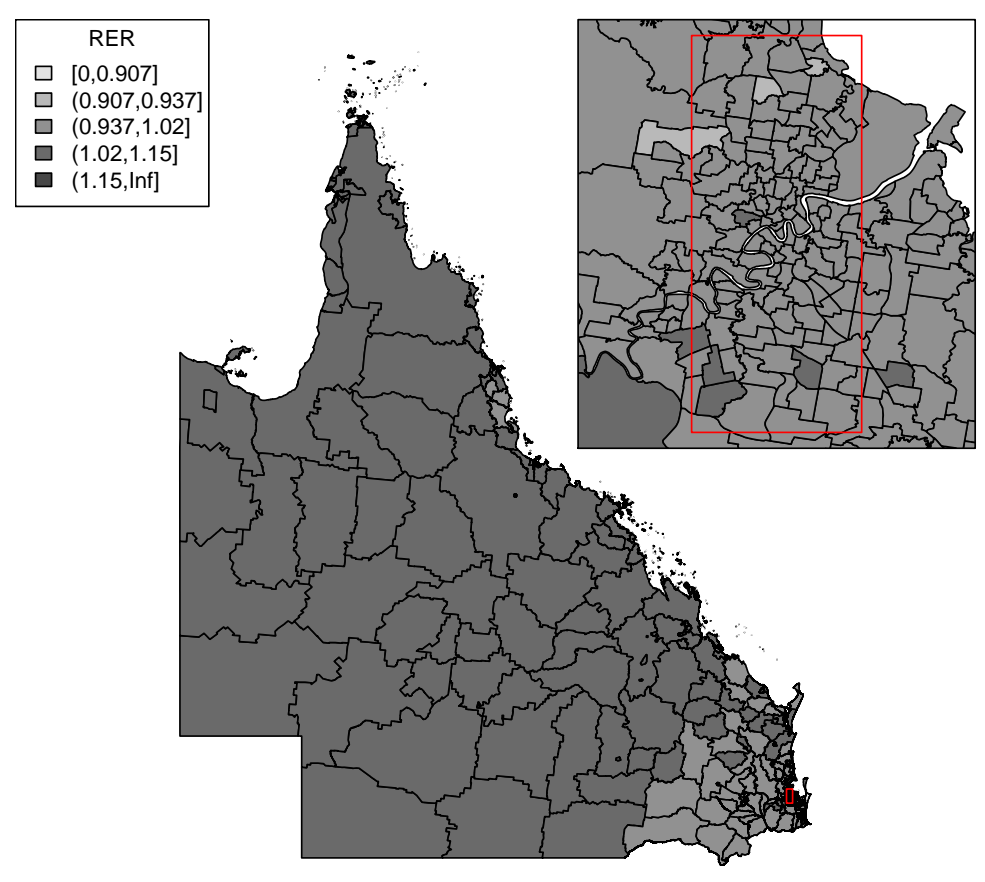

Figure 2: Posterior estimates of RER for each SLA in Queensland \& the southeast corner, based on Model M2_3

In addition, our finding that breast cancers diagnosed through population-based mammography screening have a two-fold survival advantage is consistent with the direction and strength of the effect reported by those case control studies that have used mortality as the end-point. A recent study in Western Australia and meta analysis of mammographic screening and breast cancer mortality (Nickson et al., 2012) reported that there was a $49 \%$ reduction in breast cancer mortality for women who were screened. This reduction was consistent across a range of studies covering five countries (Palli et al., 1989; Miltenburg et al., 1998; Fielder et al., 2004; Gabe et al., 2007; Allgood et al., 2008; Puliti et al., 2008; Roder et al., 2008; van Schoor et al., 2011; Otto et al., 2012).

However we found no evidence that the strong and beneficial impact of screening on breast cancer survival outcomes varied by geographic location for area disadvantage or remoteness. In contrast to the reported lower rates of PSA testing for prostate cancer among men living in rural areas of Australia (Baade et al., 2011b), breast screening participation rates have been reported to be slightly higher in rural and remote areas than in urban areas (Youlden et al., 2009). These results are encouraging, and reinforce the priority given to rural and remote areas of the state by BreastScreen 


\section{BAYESIAN SPATIAL MODEL FOR BREAST CANCER}

Queensland to offset the barrier of distance by providing mobile screening vans, in addition to re-locatable and satellite services.

The impact that socio-demographic factors had on breast cancer survival in our study was consistent with that reported previously in a multilevel analysis of breast cancer survival in Queensland (Dasgupta et al., 2012) and other related studies (Condon et al., 2005; Shen et al., 2005, 2007; Wishart et al., 2008; Cramb et al., 2011a, 2012). That these independent associations held within a different methodological framework reinforce the need to identify ways to reduce the survival inequalities, particularly among indigenous women, older women and those who are not married. The estimated RER for age at diagnosis varied slightly when method of detection was added into the analysis, with poorer survival among younger women reversing to better survival after adjustment. One explanation of this could be that these cancers are generally symptomatic (Anders et al., 2008; Yankaskas, 2006; Axelrod et al., 2008; Baade et al., 2011a) and not detected via screening (since the target age for breast screening is 50-69 years). This explanation is reinforced by the results, in that when the effect of screening is taken into account, younger women actually have slightly better adjusted survival.

One of the unique features of the Bayesian spatial methodology is the use of spatial random effects to accommodate possible spatial variation in the data. The majority of research investigating breast cancer screening has ignored the spatial location of cases, and the presence of any spatial correlation (Shen et al., 2005; Berry et al., 2006; Wishart et al., 2008; Mook et al., 2011; Nagtegaal et al., 2011; Bell \& Burton, 2012; Roder \& Olver, 2012). Inclusion of the random effect components enables a more realistic estimate of the RER in each SLA location and a better model fit (Besag et al., 1991; Best et al., 2005; Saez et al., 2012). Information from the random effect components can also be used to quantify and understand the spatial variation within the model, and how this varies between models. The disease maps also facilitate the visualization of spatial survival inequalities across the study regions, after adjusting for different health-related factors (Cramb et al., 2012).

Strengths of this study include the use of a large, unselected, population-based cohort of Queensland females diagnosed with breast cancer, with corresponding information about screening practices obtained from prospectively collected administrative data, the collection of which was designed independently of the study, thus removing information bias. The Bayesian spatial relative survival models were able to accommodate the sparse area-specific data by borrowing information from neighbouring regions to smooth the estimated spatial random effects and directly quantify the estimate of spatial variation. By focusing on relative survival we were not constrained by any potential inaccuracies in the cause of death information.

We were not able to obtain information about mammography screening offered by private hospitals and practices. However the proportion of private mammography screens has been estimated to be very low, at between 1-4\% (Australian Government Department of Health and Ageing, 2012; Nickson et al., 2012). Therefore, by placing the privately screened women in the unscreened group, the true screening differential should not be badly underestimated. 


\section{BAYESIAN SPATIAL MODEL FOR BREAST CANCER}

This study has demonstrated a clear survival benefit in respect of breast cancers detected through mammographic screening among women aged less than 90 years at diagnosis, and that this benefit was consistent after adjusting for spread of disease and diagnosis. We found no substantive evidence that the screening benefit varied according to geographic location of residence. However since women diagnosed with breast cancer while living in rural and remote areas of Queensland are at higher risk of being diagnosed with advanced disease (Baade et al., 2011a), and since this explains much of the poorer survival outcomes in these areas (Dasgupta et al., 2012; Cramb et al., 2012) there remains an urgent need to increase screening and treatment participation among women in rural, remote and disadvantaged areas to reduce the current survival inequalities.

\section{References}

AIHW (2004) Rural, regional and remote health: a guide to remoteness classifications. No. Cat. No. PHE 53. Canberra: AIHW.

AIHW \& AACR (2012) Cancer in Australia: an overview, 2012. Cancer series no. 74. Cat. no. CAN 70. Canberra: AIHW.

AIHW \& NBCC (2007) Breast cancer survival by size and nodal status in Australia. In Cancer series no. 39, no. Cat. no. CAN 34. Canberra: AIHW.

Albert, A., Gertman, P. M., Louis, T. A. \& Liu, S. (1978) Screening for the early detection of cancer ii. the impact of screening on the natural history of the disease. Math Biosci, 40, 61-109.

Allgood, P. C., Warwick, J., Warren, R. M. L., Day, N. E. \& Duffy, S. W. (2008) A casecontrol study of the impact of the East Anglian breast screening programme on breast cancer mortality. Br J Cancer, 98, 206-209.

Anders, C. K., Hsu, D. S., Broadwater, G., Acharya, C. R., Foekens, J. A., Zhang, Y., Wang, Y., Marcom, P. K., Marks, J. R., Febbo, P. G., Nevins, J. R., Potti, A. \& Blackwell, K. L. (2008) Young age at diagnosis correlates with worse prognosis and defines a subset of breast cancers with shared patterns of gene expression. $J$ Clin Oncol, 26, 3324-3330.

Australian Bureau of Statistics (2008) Census of Population and Housing: SocioEconomic Indexes for Areas (SEIFA), Australia, 2006. ABS Cat. No. 2033.0.55.001. ABS, Canberra.

Australian Government Department of Health and Ageing (2012) BreastScreen Program. BreastScreen Australia Evaluation. [cited 2012 Sep 12]. Available from: http://www.cancerscreening.gov.au/internet/screening/publishing.nsf/Content/brevaluation-lp. 


\section{BAYESIAN SPATIAL MODEL FOR BREAST CANCER}

Axelrod, D., Smith, J., Kornreich, D., Grinstead, E., Singh, B., Cangiarella, J. \& Guth, A. (2008) Breast cancer in young women. J Am Coll Surg, 206, 1193-203.

Baade, P., Turrell, G. \& Aitken, J. (2011a) Geographic remoteness, area-level socioeconomic disadvantage and advanced breast cancer: a cross-sectional, multilevel study. J Epidemiol Community Health, 65, 1037-1043.

Baade, P. D., Youlden, D. R., Coory, M. D., Gardiner, R. A. \& Chambers, S. K. (2011b) Urbanrural differences in prostate cancer outcomes in Australia: what has changed? Med J Aust, 194(6), 293-296.

Banerjee, S., Wall, M. \& Carlin, B. (2003) Frailty modeling for spatially correlated survival data, with application to infant mortality in minnesota. Biostatistics, 4, $123-142$.

Barratt, A. L. \& Glasziou, P. P. (2012) Do the benefits of screening mammography outweigh the harms of overdiagnosis and unnecessary treatment? Med J Aust, 196 (11), 681 .

Bell, B. S. \& Broemeling, L. D. (2000) A Bayesian analysis for spatial processes with application to disease mapping. Stat Med, 19, 957-974.

Bell, R. J. \& Burton, R. C. (2012) Do the benefits of screening mammography outweigh the harms of overdiagnosis and unnecessary treatment? - No. Med J Aust, $196(1), 17$.

Berry, D., Cronin, K., Plevritis, S., Fryback, D., Clarke, L., Zelen, M., Mandelblatt, J., Yakovlev, A., Habbema, J. \& Feuer, E. (2005) Effect of screening and adjuvant therapy on mortality from breast cancer. N Engl J Med, 353, 1784-1792.

Berry, D., Inoue, L., Shen, Y., Venier, J., Cohen, D., Bondy, M., Theriault, R. \& Munsell, M. (2006) Modeling the impact of treatment and screening on US breast cancer mortality: a Bayesian approach. J Natl Cancer Inst Monogr, 2006, 30-36.

Besag, J., York, J. \& Molli, A. (1991) Bayesian image restoration, with two applications in spatial statistics. Ann Inst Statist Math, 43, 1-20.

Best, N., Richardson, S. \& Thomson, A. (2005) A comparison of Bayesian spatial models for disease mapping. Stat Methods Med Res, 14, 35-59.

Bordas, P., Jonsson, H., Nystrm, L. \& Lenner, P. (2007) Survival from invasive breast cancer among interval cases in the mammography screening programmes of northern sweden. The Breast, 16, 47-54.

Chu, K. C., Smart, C. R. \& Tarone, R. E. (1988) Analysis of breast cancer mortality and stage distribution by age for the health insurance plan clinical trial. $J$ Natl Cancer Inst, 80, 1125-1132. 


\section{BAYESIAN SPATIAL MODEL FOR BREAST CANCER}

Condon, J., Barnes, T., Armstrong, B. \& et al. (2005) Stage at diagnosis and cancer survival for indigenous Australians in the Northern Territory. Med J Aust, 182, $277-80$.

Connor, R. J., Chu, K. C. \& Smart, C. R. (1989) Stage-shift cancer screening model. J Clin Epidemiol, 42, 1083-1095.

Cramb, S., Mengersen, K. \& Baade, P. (2011a) Atlas of Cancer in Queensland: geographical variation in incidence and survival, 1998 to 200\%. Viertel Centre for Research in Cancer Control, Cancer Council Queensland.

Cramb, S. M., Mengersen, K. L. \& Baade, P. D. (2011b) Developing the atlas of cancer in Queensland: Methodological issues. Int J Health Geogr, 10, 9.

Cramb, S. M., Mengersen, K. L., Turrell, G. \& Baade, P. D. (2012) Spatial inequalities in colorectal and breast cancer survival: Premature deaths and associated factors. Health Place, 18, 1412-1421. [in press, available online].

Dasgupta, P., Baade, P., Aitken, J. \& Turrell, G. (2012) Multilevel determinants of breast cancer survival: association with geographic remoteness and area-level socioeconomic disadvantage. Breast Cancer Res Treat, 132(2), 701-710.

Dickman, P., Sloggett, A., Hills, M. \& Hakulinen, T. (2004) Regression models for relative survival. Stat $M e d, \mathbf{2 3}, 51-64$.

Earnest, A., Morgan, G., Mengersen, K., Ryan, L., Summerhayes, R. \& Beard, J. (2007) Evaluating the effect of neighbourhood weight matrices on smoothing properties of conditional autoregressive (CAR) models. Int $J$ Health Geogr, 6, 54.

Eberly, L. E. \& Carlin, B. P. (2000) Identifiability and convergence issues for Markov chain Monte Carlo fitting of spatial models. Stat Med, 19, 2279-2294.

Fairley, L., Forman, D., West, R. \& Manda, S. (2008) Spatial variation in prostate cancer survival in the Northern and Yorkshire region of England using Bayesian relative survival smoothing. Br J Cancer, 99, 1786-1793.

Fielder, H., Warwick, J., Brook, D., Gower-Thomas, K., Cuzick, J., Monypenny, I. \& Duffy, S. (2004) A case-control study to estimate the impact on breast cancer death of the breast screening programme in Wales. J Med Screen, 11, 194-198.

Gabe, R., Tryggvadottir, L., Sigfusson, B. F., Olafsdottir, G. H., Sigurdsson, K. \& Duffy, S. W. (2007) A Case-Control Study to Estimate the Impact of the Icelandic Population-Based Mammography Screening Program on Breast Cancer Death. Acta Radiol, 48, 948-955.

Gelman, A., Carlin, J., Stern, H. \& Rubin, D. (2004) Bayesian data analysis. Chapman \& Hall/CRC. 


\section{BAYESIAN SPATIAL MODEL FOR BREAST CANCER}

Gelman, A. \& Rubin, D. (1992) Inference from iterative simulation using multiple sequences. Statist Sci, 7, 457-472.

Ghosh, M., Natarajan, K., Waller, L. A. \& Kim, D. (1999) Hierarchical Bayes GLMs for the analysis of spatial data: An application to disease mapping. J Statist Plann Inference, $75,305-318$.

Hegarty, A., Carsin, A. \& Comber, H. (2010) Geographical analysis of cancer incidence in Ireland: A comparison of two Bayesian spatial models. Cancer Epidemiol, 34, 373-381.

Huang, B., Dignan, M., Han, D. \& Johnson, O. (2009) Does Distance Matter? Distance to Mammography Facilities and Stage at Diagnosis of Breast Cancer in Kentucky. J Rural Health, 25, 366-371.

Joensuu, H., Lehtimki, T., Holli, K., Elomaa, L., Turpeenniemi-Hujanen, T., V, K. \& et al. (2004) Risk for distant recurrence of breast cancer detected by mammography screening or other methods. JAMA, 292, 1064-1073.

Kavanagh, A., Amos, A. F. \& Mark, G. M. (1999) The Ascertainment and Reporting of Interval Cancers Within the Breast Screen Australian Program. Sydney: NHMRC NBCC.

Kramer, B., Gohagan, J. \& Prorok, P. (1999) Cancer screening: theory and practice. New York (NY): Marcel Dekker.

Lawson, A., Biggeri, A., Boehning, D., Lesaffre, E., Viel, J., Clark, A., Schlattmann, P. \& Divino, F. (2000) Disease mapping models: an empirical evaluation. Disease Mapping Collaborative Group. Stat Med, 19, 2217-41.

Lawson, A. B. (2001) Disease map reconstruction. Stat Med, 20, 2183-2204.

Lunn, D., Thomas, A., Best, N. \& Spiegelhalter, D. (2000) WinBUGS - a Bayesian modelling framework: concepts, structure, and extensibility. Stat Comput, 10, 325-337.

MacNab, Y. (2004) Bayesian spatial and ecological models for small-area accident and injury analysis. Accid Anal Prev, 36, 1019-1028.

MacNab, Y. \& Dean, C. (2002) Spatio-temporal modelling of rates for the construction of disease maps. Stat Med, 21, 347-358.

McPherson, C., Swenson, K., Jolitz, G. \& Murray, C. (1997) Survival of women ages 40-49 years with breast carcinoma according to method of detection. Cancer, 79, 1923-1932.

Militino, A. F., Ugarte, M. D. \& Dean, C. B. (2001) The use of mixture models for identifying high risks in disease mapping. Stat Med, 20, 2035-2049. 


\section{BAYESIAN SPATIAL MODEL FOR BREAST CANCER}

Miltenburg, G. A., Peeters, P. H., Fracheboud, J., \& Collette, H. J. (1998) Seventeenyear evaluation of breast cancer screening: the DOM project, The Netherlands. Diagnostisch Onderzoek (investigation) Mammacarcinoom. Br J Cancer, 78(7), 962-965.

Mook, S., Veer, L. V., Rutgers, E., Ravdin, P., van de Velde, A., van Leeuwen, F., Visser, O. \& Schmidt, M. (2011) Independent prognostic value of screen detection in invasive breast cancer. J Natl Cancer Inst, 103, 585-597.

Morrison, A. S. (1985) Screening in chronic disease, vol. 7. New York (NY): Oxford University Press.

Nagtegaal, I., Allgood, P., Duffy, S., Kearins, O., Sullivan, E., Tappenden, N., Wallis, M. \& Lawrence, G. (2011) Prognosis and pathology of screen detected carcinomas. Cancer, 117, 1360-1368.

Nickson, C., Mason, K. E., English, D. R. \& Kavanagh, A. M. (2012) Mammographic Screening and Breast Cancer Mortality: A Case-Control Study and Meta-analysis. Cancer Epidemiol Biomarkers Prev, 21, 1479-1488.

Olsen, O. \& Gtzsche, P. (2001) Cochrane review on screening for breast cancer with mammography. The Lancet, 358, 1340-1342.

Osnes, K. \& Aalen, O. (1999) Spatial smoothing of cancer survival: a Bayesian approach. Stat Med, 18, 2087-2099.

Otto, S., Fracheboud, J., Looman, C., Broeders, M., Boer, R., Hendriks, J. \& et al (2003) Initiation of population-based mammography screening in dutch municipalities and effect on breast-cancer mortality: a systematic review. Lancet, 361, $1411-7$.

Otto, S. J., Fracheboud, J., Verbeek, A. L., Boer, R. \& et al (2012) Mammography Screening and Risk of Breast Cancer Death: A Population-Based CaseControl Study. Cancer Epidemiol Biomarkers Prev, 21, 66-73.

Palli, D., del Turco, M. R., Buiatti, E., Ciatto, S., Crocetti, E. \& Paci, E. (1989) Time interval since last test in a breast cancer screening programme: a case-control study in Italy. J Epidemiol Community Health, 43, 241-248.

Pohar, M. \& Stare, J. (2006) Relative survival analysis in R. Comput Methods Programs Biomed, 81, 272-278.

Puigpins-Riera, R., Mar-Dell'Olmo, M., Gotsens, M., Borrell, C., Serral, G., Ascaso, C., Calvo, M., Daponte, A., Domnguez-Berjn, F. \& Esnaola, S. (2011) Cancer mortality inequalities in urban areas: a Bayesian small area analysis in Spanish cities. Int J Health Geogr, 10, 6. 


\section{BAYESIAN SPATIAL MODEL FOR BREAST CANCER}

Puliti, D., Miccinesi, G., Collina, N., Lisi, V. D., Federico, M., Ferretti, S. \& et al (2008) Effectiveness of service screening: a case-control study to assess breast cancer mortality reduction. Br J Cancer, 99, 423-427.

R Development Core Team (2011) R: A Language and Environment for Statistical Computing. R Foundation for Statistical Computing, Vienna, Austria. URL http://www.R-project.org/. ISBN 3-900051-07-0.

Richardson, S., Abellan, J. \& Best, N. (2006) Bayesian spatio-temporal analysis of joint patterns of male and female lung cancer risks in Yorkshire (UK). Stat Methods Med Res, 15, 385-407.

Robsahm, T. \& Tretli, S. (2008) Weak associations between sociodemographic factors and breast cancer: possible effects of early detection. Eur J Cancer Prev, 14, 7-12.

Roder, D., Houssami, N., Farshid, G., Gill, G., Luke, C., Downey, P. \& et al (2008) Population screening and intensity of screening are associated with reduced breast cancer mortality: evidence of efficacy of mammography screening in Australia. Breast Cancer Res Treat, 108, 409-416.

Roder, D. M. \& Olver, I. N. (2012) Do the benefits of screening mammography outweigh the harms of overdiagnosis and unnecessary treatment? - Yes. Med $J$ Aust, 196 (1), 16.

Saez, M., Barcel, M., Martos, C., Saurina, C., Marcos-Gragera, R., Renart, G., OcaaRiola, R., Feja, C. \& Alcal, T. (2012) Spatial variability in relative survival from female breast cancer. J Roy Statist Soc Ser A, 175, 107-134.

Sariego, J. (2009) Patterns of breast cancer presentation in the United States: does geography matter? Am Surg, 75, 545-550(6).

van Schoor, G., Moss, S. M., Otten, J. D. M., Donders, R., Paap, E., den Heeten, G. J. \& et al (2011) Increasingly strong reduction in breast cancer mortality due to screening. Br J Cancer, 104, 910-914.

Shen, Y., Dong, W., Esteva, F., Kau, S., Theriault, R. \& Bevers, T. (2007) Are there racial differences in breast cancer treatments and clinical outcomes for women treated at MD Anderson Cancer Center? Breast Cancer Res Treat, 102, 347-356.

Shen, Y., Yang, Y., Inoue, L., Munsell, M., Miller, A. \& Berry, D. (2005) Role of detection method in predicting breast cancer survival: analysis of randomized screening trials. J Natl Cancer Inst, 97, 1195-1203.

Tabar, L., Yen, M., Vitak, B., Chen, H., Smith, R. \& Duffy, S. (2003) Mammography service screening and mortality in breast cancer patients: 20-year follow-up before and after introduction of screening. The Lancet, 361, 1405-1410.

Wakefield, J. (2007) Disease mapping and spatial regression with count data. Biostatistics, 8, 158-183. 
Waller, L., Carlin, B., Xia, H. \& Gelfand, A. (1997) Hierarchical spatio-temporal mapping of disease rates. J Amer Statist Assoc, 607-617.

Wishart, G., Greenberg, D., Britton, P., Chou, P., Brown, C., Purushotham, A. \& Duffy, S. (2008) Screen-detected vs symptomatic breast cancer: is improved survival due to stage migration alone? Br J Cancer, 98, 1741-1744.

Wu, J., Anttila, A., Yen, A., Hakama, M., Saarenmaa, I., Sarkeala, T., Malila, N., Auvinen, A., Chiu, S. \& Chen, T. (2010) Evaluation of breast cancer service screening programme with a Bayesian approach: mortality analysis in a Finnish region. Breast Cancer Res Treat, 121, 671-678.

Yankaskas, B. C. (2006) Epidemiology of breast cancer in young women. Breast Dis, 23, 3-8.

Youlden, D., Cramb, S. \& Baade, P. (2009) Current status of female breast cancer in Queensland, 1982 to 2006. Viertel Centre for Research in Cancer Control, Cancer Council Queensland. 